\title{
Tell Me a Pseudo-Anosov
}

Erwan Lanneau (Université Grenoble Alpes, Saint-Martin-d'Hères, France)

Anosov linear homeomorphisms, and more generally Anosov flows, as well as their hyperbolic analogues, have played an important role in the theory of dynamical systems $[1,2,7] .{ }^{1}$

Their cousins, the pseudo-Anosov homeomorphisms, which are also interesting and important, seem to be less well known. In contrast to the theory of Anosov flows, for which we know their contours rather well, there are several fundamental questions about pseudo-Anosov homeomorphisms that so far remain widely open.

\section{$1 \quad$ An instructive example}

Let us start with a simple example that is, in some sense, more than an example. Any matrix $A \in \operatorname{SL}(2, \mathbb{Z})$ acts linearly on the plane $\mathbb{R}^{2}$. The induced dynamics are not very interesting (the orbits are either circles or escape to infinity). A method of making things richer is to "pass to the quotient": since $A$ bijectively preserves the $\mathbb{Z}^{2}$ lattice, that is, $A\left(\mathbb{Z}^{2}\right)=\mathbb{Z}^{2}$, it induces a diffeomorphism $\psi$ of the torus $T^{2}=\mathbb{R}^{2} / \mathbb{Z}^{2}$ given by $\psi\left((x, y)+\mathbb{Z}^{2}\right)=A(x, y)+\mathbb{Z}^{2}$.

The dynamics of $\psi$ are governed by the eigenvalues $\lambda, \lambda^{-1}$ of $A$. There are three possibilities:

(1) $\lambda$ and $\lambda^{-1}$ are complex conjugates $(\lambda \neq \pm 1): \psi$ is of finite order.

(2) $\lambda=\lambda^{-1}= \pm 1: \psi$ is reducible, that is, it preserves a closed curve on the torus.

(3) $\lambda$ and $\lambda^{-1}$ are distinct irrational numbers: $\psi$ is of Anosov type.

The second case (parabolic) implies that $\psi$ arises from a map on a simpler surface (in this case an annulus).

The last case (hyperbolic) is by far the one with the richer dynamics ( $\psi$ has many periodic points, many points of dense orbits, etc.). The cat map $A=\left(\begin{array}{ll}2 & 1 \\ 1 & 1\end{array}\right)$, for which $\lambda=(3+\sqrt{5}) / 2$, is a nice illustration of this situation (see [5]). These maps, although very simple, capture many properties of elements in an open subset of the set of diffeomorphisms of the torus $T^{2}$ : this is the famous Anosov [1] result on structural stability. It states that any diffeomorphism $\phi$ sufficiently close to an hyperbolic diffeomorphism $\psi$ in the $C^{1}$ topology is topologically conjugated to $\psi$ : there exists a homeomorphism $h \in \operatorname{Homeo}\left(T^{2}\right)$ such that $\phi=h \circ \psi \circ h^{-1}$. Hence, $\phi$ and $\psi$ are the same up to a change of coordinates.

Thus, these Anosov diffeomorphisms provide important information on large open subsets of the group $\operatorname{Diff}^{+}\left(T^{2}\right)$. Their hyperbolic counterparts have occupied mathematicians since then: they are the main actors of $\operatorname{Diff}^{+}\left(S_{g}\right)$, the group of diffeomorphisms of a genus $g$ surface $S_{g}$.

These Anosov diffeomorphisms are so important that they are also the actors of another family of groups: the modular

1 See the article by A. Bufetov and A. Klimenko in the Gazette des Mathématiciens (No. 143, January 2015). groups. In the 1970s, Thurston [8] generalised the analysis carried out on a torus to the case of compact surfaces, thus extending the notion of Anosov maps to that of pseudo-Anosov maps.

\section{Foliations and pseudo-Anosov homeomorphisms}

\section{Measured foliation}

An important feature of a linear Anosov of the torus is that it leaves invariant the two foliations $\mathcal{F}^{u}$ and $\mathcal{F}^{s}$ of "straight lines" of constant slopes (parallel to the directions of the eigenvectors associated to $\lambda$ and $\lambda^{-1}$ ). These foliations also come with an additional structure: they are integrable in the sense that we can define them globally as the kernel of a closed 1-form $d v$.

Hence, we have a measure $\mu_{s}$ defined on arcs $\alpha$ transverse to the leaves of $\mathcal{F}^{s}$, measuring the total variation of $\alpha$ in the orthogonal direction: $\mu_{s}(\alpha)=\int_{\alpha} d v_{s}$.

The measure is invariant in the sense that if we change the extremities of $\alpha$ in the same leaf, the measure remains unchanged. The data $\left(\mathcal{F}^{s}, \mu_{s}\right)$ is a measured foliation. Of course, our Anosov preserves these leaves and expands/contracts the measures: we can think that $\psi$ expands by a factor $\lambda$ in the direction of $\mathcal{F}^{u}$ and contracts by the same factor in the direction of $\mathcal{F}^{s}$.

On a surface of higher genus, the notion of measured foliations also exists but the Gauß-Bonnet formula forces us to extend them to singular foliations. For pairs of transverse measured foliations there is a very elegant way of doing this with the help of half-translation structures.

If $\Sigma \subset S_{g}$ is a finite set, a half-translation structure on $\left(S_{g}, \Sigma\right)$ is an atlas of charts $\omega=\left(U_{\alpha}, z_{\alpha}\right)$ of $S \backslash \Sigma$ for which the changes of charts are of the form $z \mapsto \pm z+$ const and such that each point of $\Sigma$ has a neighbourhood isometric to a finite cover of $\mathbb{R}^{2} \backslash\{0\}$. The pullback of the horizontal and vertical leaves of $\mathbb{R}^{2}$ thus defines a pair of transverse measured foliations on $S_{g}$ (the measures are $d y$ and $d x$ respectively).

Example 2.1. Figure 1 represents, on the left, a half-translation structure on the surface $S_{2}$ : we glue together the sides with the same labels. We can verify that the vertices of the $L$ shaped polygon represent a single point in $S_{2}$, which is singular. It has two obvious measured foliations (horizontal and vertical) with transverse measures $d y$ and $d x$ respectively.

Warning! There are measured foliations that do not arise from this construction (and so do not admit a transverse measured foliation). In the following example (following Hubbard-Masur), we glue two cylinders, foliated by circles, according to Figure 2: the boundaries of the first cylinder are the arcs $\gamma_{1}, \gamma_{2}$ and $\gamma_{1}, \gamma_{3}, \gamma_{4}, \gamma_{6}$ and those of the second cylinder are $\gamma_{5}, \gamma_{6}$ and $\gamma_{2}, \gamma_{3}, \gamma_{4}, \gamma_{5}$. The transverse measure 


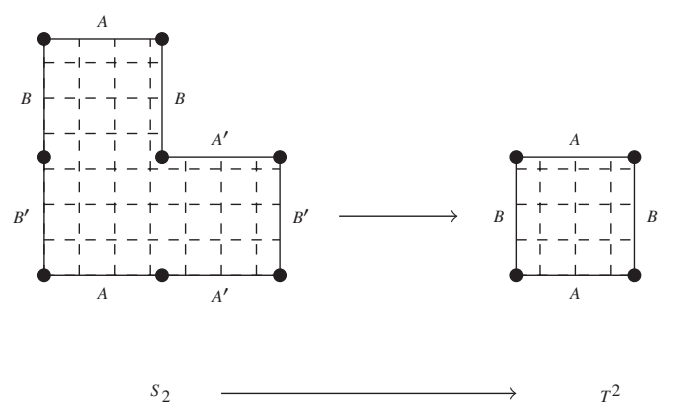

Figure 1. Triple cover of the standard torus: surface with three tiles

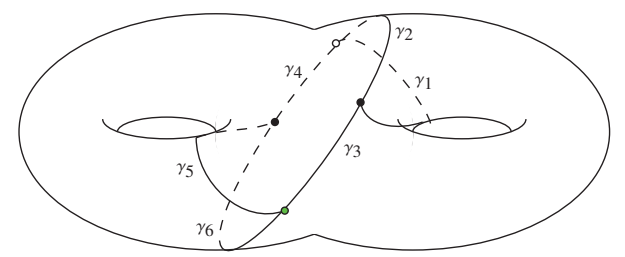

Figure 2. Measured foliation on a surface of genus two with four singularities (according to Hubbard-Masur)

is given by the "height function". We can observe that a transverse foliation does not exist, otherwise the cylinders would have boundaries with equal lengths. This does not occur since the linear system

$$
\left\{\begin{array}{l}
\left|\gamma_{1}\right|+\left|\gamma_{2}\right|=\left|\gamma_{1}\right|+\left|\gamma_{3}\right|+\left|\gamma_{4}\right|+\left|\gamma_{6}\right| \\
\left|\gamma_{5}\right|+\left|\gamma_{6}\right|=\left|\gamma_{2}\right|+\left|\gamma_{3}\right|+\left|\gamma_{4}\right|+\left|\gamma_{5}\right|
\end{array}\right.
$$

does not admit any strictly positive solutions.

\section{Pseudo-Anosov homeomorphisms}

A homeomorphism $\psi: S \rightarrow S$ is a pseudo-Anosov homeomorphism if there exist a pair of measured transverse foliations $\left(\mathcal{F}^{u}, \mu_{u}\right)$ and $\left(\mathcal{F}^{s}, \mu_{s}\right)$ on $S_{g}$, called unstable and stable respectively, and a number $\lambda>1$ (the expansion factor of $\psi$ ) such that

$$
\begin{aligned}
& \psi \cdot\left(\mathcal{F}^{u}, \mu_{u}\right)=\left(\mathcal{F}^{u}, \lambda \cdot \mu_{u}\right), \quad \text { and } \\
& \psi \cdot\left(\mathcal{F}^{s}, \mu_{s}\right)=\left(\mathcal{F}^{s}, \lambda^{-1} \cdot \mu_{s}\right) .
\end{aligned}
$$

An equivalent way to formulate this is to say that $\psi$ is an affine diffeomorphism on $S \backslash \Sigma$ for the Euclidian metric defined above and that its differential $D \psi=\left(\begin{array}{cc} \pm \lambda & 0 \\ 0 & \pm \lambda^{-1}\end{array}\right)$ is hyperbolic, that is, $|\operatorname{tr}(D \psi)|>2$ (in general, $\psi$ is not differentiable at the points of $\Sigma$ ). The group formed by all differentials $D \psi$ with $\psi$ affine for the atlas $\omega$ is called the Veech group $\operatorname{SL}(S, \omega) \subset \operatorname{PSL}(2, \mathbb{R})$.

Although rather natural, it is not an easy task to construct examples satisfying this definition (at least in genus different from 1). A way of achieving it is to lift linear Anosov maps on the torus to coverings.

Example 2.2. The linear Anosov on the torus $\psi: T^{2} \rightarrow T^{2}$, with differential $A=\left(\begin{array}{ll}5 & 2 \\ 2 & 1\end{array}\right)=\left(\begin{array}{ll}1 & 2 \\ 0 & 1\end{array}\right)\left(\begin{array}{ll}1 & 0 \\ 2 & 1\end{array}\right)$, lifts (see Example 2.1) to a pseudo-Anosov $\widetilde{\psi}: S_{2} \rightarrow S_{2}$ such that $\bar{D} \widetilde{\psi}=A$, as will be explained in Section 4.

\section{$3 \quad$ Modular group}

The pseudo-Anosov homeomorphisms are the elementary building blocks for the study of modular groups of surfaces. The group in question is always $\operatorname{Difff}^{+}\left(S_{g}\right)$ but this time up to continuous deformation (we shall say up to isotopy). More precisely, the modular group is the quotient group $\operatorname{Diff}^{+}\left(S_{g}\right)$ by the group $\operatorname{Diff}\left(S_{g}\right)_{0}$ of diffeomorphisms isotopic to the identity: $\operatorname{Mod}\left(S_{g}\right)=\operatorname{Diff}^{+}\left(S_{g}\right) / \operatorname{Diff}\left(S_{g}\right)_{0}$.

Sometimes, definitions differ from one source to another, e.g., group of diffeomorphisms or group of homeomorphisms. It does not matter: the quotient groups are all isomorphic (even if the groups $\operatorname{Diff}^{+}\left(S_{g}\right)$ and $\operatorname{Homeo}^{+}\left(S_{g}\right)$ are very different!).

\section{Nielsen-Thurston classification}

We are now able to state the classification theorem of surface homeomorphisms, which is very close to the one on the torus. Any $f \in \operatorname{Homeo}^{+}\left(S_{g}\right)$ is, up to isotopy, either:

(1) Periodic: there exists $m$ such that $f^{m}=$ Id.

(2) Reducible: $f$ preserves a family of simple closed curves.

(3) A pseudo-Anosov map.

In the second case, some iterate of $f$ preserves a subsurface (with boundaries). As we can again apply the theorem to this subsurface, the third case is by far the most interesting one!

\section{Classical modular groups}

The modular group of the closed disc is rather simple to describe (here our surface has a boundary: we require the homeomorphism to be the identity map on the boundary).

Such a map $\phi$ defined on $\overline{D(0,1)}$ can easily be deformed by an isotopy acting like $\phi$ on the small disc of radius $t<1$ and being the identity outside. In coordinates, this is

$$
F(z, t)=\left\{\begin{array}{l}
t \phi(z / t), \text { if } z \in D(0, t) \text { and } t \neq 0 \\
z, \text { otherwise. }
\end{array}\right.
$$

We have $F(\cdot, 0)=$ Id and $F(\cdot, 1)=\psi$. With this idea we easily prove that the modular groups of the disc and of the sphere are trivial.

Although somewhat simplistic, this approach is fundamental: Magnus remarked in 1934 that the action of isotopies on the punctures allows the connection of two a priori distinct groups: the modular group on the disc with $n$ punctures and the braid group on $n$ strands.

The first nontrivial example of a modular group is the one of the flat cylinder $C$. If $\gamma$ is an oriented, simple closed curve linking the two components of the boundary of $C$ then the homeomorphism $T_{\gamma}$ that twists the cylinder along $\gamma$ is nontrivial in $\operatorname{Mod}(C)=\left\langle T_{\gamma}\right\rangle \simeq \mathbb{Z}$. The homeomorphism $T_{\gamma}$ has a very simple expression in the parametrisation $C=\mathbb{R} / w \mathbb{Z} \times[0 ; h]$ :

$$
T_{\gamma}(x, y)=(x+w / h \cdot y, y)=\left(x+\mu^{-1} y, y\right),
$$

where $\mu=h / w$ is the modulus of the cylinder $C$. It is actually a diffeomorphism and $D T_{\gamma}=\left(\begin{array}{ll}1 & \mu^{-1} \\ 0 & 1\end{array}\right)$.

Furthermore, since any surface $S_{g}$ contains an annulus $C$, we can define by analogy $T_{\gamma} \in \operatorname{Mod}\left(S_{g}\right)$ along a simple closed curve $\gamma$ (since $T_{\gamma}$ is the identity on the boundary of the annulus). These elements take an important place in the study of the modular group: we call them Dehn twists. 


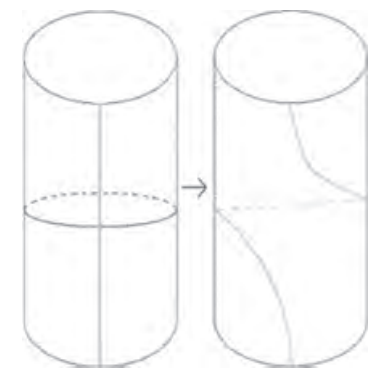

Figure 3. Dehn twist along a curve

\section{Modular group of the torus}

Writing $T^{2}=\mathbb{R} / \mathbb{Z} \times \mathbb{R} / \mathbb{Z}$, we can define two Dehn twists along the two curves $\alpha=(1,0)$ and $\beta=(0,1)$ : this provides a "large" subgroup of $\operatorname{Mod}\left(T^{2}\right):\left\langle T_{\alpha}, T_{\gamma}\right\rangle=\left\langle\left(\begin{array}{ll}1 & 1 \\ 0 & 1\end{array}\right),\left(\begin{array}{ll}1 & 0 \\ 1 & 1\end{array}\right)\right\rangle=$ $\mathrm{SL}(2, \mathbb{Z})$ (we identify here a Dehn twist with its differential).

In fact, by letting a homeomorphism of $T^{2}$ act on the homology $H_{1}\left(T^{2}, \mathbb{Z}\right)=\langle\alpha, \beta\rangle \simeq \mathbb{Z}^{2}$, we obtain an isomorphism

$$
\operatorname{Mod}\left(T^{2}\right) \simeq \operatorname{SL}(2, \mathbb{Z})=\operatorname{Aut}\left(\mathbb{Z}^{2}\right)
$$

that provides us with a rather precise description of the modular group of genus one surfaces.

\section{Modular group of a surface}

Just as we understand $\operatorname{Mod}\left(T^{2}\right)$ with the help of action on curves, we can study $\operatorname{Mod}\left(S_{g}\right)$ through the action of $\operatorname{Diff}^{+}\left(S_{g}\right)$ on simple closed curves of $S_{g}$. This time it is more complicated than it seems because such a curve can be extremely complicated.

By letting the homeomorphisms act on the homology $H_{1}\left(S_{g}, \mathbb{Z}\right)$, we obtain a first "linear" approach of the modular group (choosing a symplectic basis for the intersection form):

$$
\operatorname{Mod}\left(S_{g}\right) \rightarrow \operatorname{Sp}(2 g, \mathbb{Z})
$$

This homeomorphism is onto (in fact, every element of $\mathrm{Sp}(2 g, \mathbb{Z})$ can be realised by a pseudo-Anosov map, even if we do not always know how to characterise those that fix an orientable measured foliation). On the other hand, if $g \geq 2$, its kernel (the Torelli group) is rather large.

We end this section with a result analogous to the well known fact that $\operatorname{SL}(n, \mathbb{Z})$ is generated by transvection matrices.

The $\operatorname{group} \operatorname{Mod}\left(S_{g}\right)$ is generated by a finite number of Dehn twists (Dehn, 1922).

The (optimal) number of generators is $2 g+1$ (Humphries, 1977).

\section{$4 \quad$ Several constructions}

It is not an easy task to construct pseudo-Anosov homeomorphisms.

Let us give a simple and fruitful idea. An affine Dehn twist $T_{\gamma}$ possesses a parabolic differential, $\left|\operatorname{tr}\left(D T_{\gamma}\right)\right|=2$. By applying the motto

"a product of parabolic elements is 'generally' an hyperbolic element",

it is possible to show, for well chosen curves $\gamma$ and $\eta$, that $\left|\operatorname{tr}\left(D T_{\gamma} T_{\eta}\right)\right|>2$, that is, $T_{\gamma} \circ T_{\eta}$ is pseudo-Anosov. This is the Thurston-Veech construction, popularised on the occasion of a talk by John Hubbard at C.I.R.M. in Marseille in 2003. Since then, this construction has sometimes been called the bouillabaisse construction.

Example 4.1. In Example 2.1, the left surface $S_{2}$ is horizontally cut along two cylinders of height 1 with cores $\alpha_{1}, \alpha_{2}$ of length 1 and 2. Thus, $D T_{\alpha_{1}}=\left(\begin{array}{ll}1 & 1 \\ 0 & 1\end{array}\right)$ and $D T_{\alpha_{2}}=\left(\begin{array}{ll}1 & 2 \\ 0 & 1\end{array}\right)$. Since each Dehn twist $T_{\alpha_{i}}$ is equal to the identity on the boundaries of the cylinders, the "multi-twist" $T_{h}=T_{\alpha_{1}}^{2} \circ T_{\alpha_{2}}$ is a diffeomorphism on $S_{2} \backslash \Sigma$ whose differential is constant and equal to $\left(\begin{array}{ll}1 & 2 \\ 0 & 1\end{array}\right)$. By symmetry reasons, the vertical multi-twist $T_{v}=T_{\beta_{1}} \circ T_{\beta_{2}}^{2}$ is also affine and has a differential equal to $\left(\begin{array}{ll}1 & 0 \\ 2 & 1\end{array}\right)$.

We then check that $D\left(T_{h} \circ T_{v}\right)=\left(\begin{array}{ll}5 & 2 \\ 2 & 1\end{array}\right)$. This is our Example 2.2, which is pseudo-Anosov!

Example 4.2 (A more subtle example). Let us consider on a genus 2 surface the multi-curves $\alpha=\left\{2 a_{1}, a_{2}, c_{1}\right\}$ and $\beta=\left\{b_{1}, b_{2}\right\}$ (represented in Figure 4). The product of the two multi-twists $T_{\alpha} \circ T_{\beta}$, where

$$
T_{\alpha}=T_{a_{1}}^{2} \circ T_{a_{2}} \circ T_{c_{1}} \quad \text { and } \quad T_{\beta}=T_{b_{1}} \circ T_{b_{2}},
$$

is an element $\psi$ of pseudo-Anosov type. Its expansion factor $\lambda(\psi)$ is the largest real root $(\simeq 1.72)$ of the polynomial $X^{4}-$ $X^{3}-X^{2}-X+1$.

This idea produces a lot of pseudo-Anosov diffeomorphisms. A beautiful theorem of A. Fathi gives a quantitative version of this motto. Let us consider a family of distinct curves (up to isotopy) $\left\{\gamma_{1}, \ldots, \gamma_{n}\right\}$ filling $S\left(S \backslash \cup_{i} \gamma_{i}\right.$ is a union of discs). Then,

$$
\begin{aligned}
\exists N \in \mathbb{N}, \forall\left(n_{1}, \ldots, n_{k}\right) \in \mathbb{Z}^{k}: \text { if }\left|n_{i}\right| \geq N, \forall i, \text { then } \\
\quad T_{\gamma_{1}} \circ \cdots \circ T_{\gamma_{k}} \text { is isotopic to a pseudo-Anosov map. }
\end{aligned}
$$

A surprising corollary is that if $\psi$ is a pseudo-Anosov and $\gamma$ is a simple closed curve then $T_{\gamma}^{n} \circ \psi$ is isotopic to a pseudoAnosov for any non-negative integer $n$, with the possible exception of at most seven consecutive values of $n$ !

There are other constructions that we do not have time to explain, which are algorithmic and, in certain cases, allow us to describe all the pseudo-Anosov maps. Here are a few of them:

(1) Train track induction.

(2) The Rauzy-Veech induction.

(3) Sections of flows on hyperbolic 3-manifolds.

The first induction has been extensively studied by Papadopoulos and Penner.

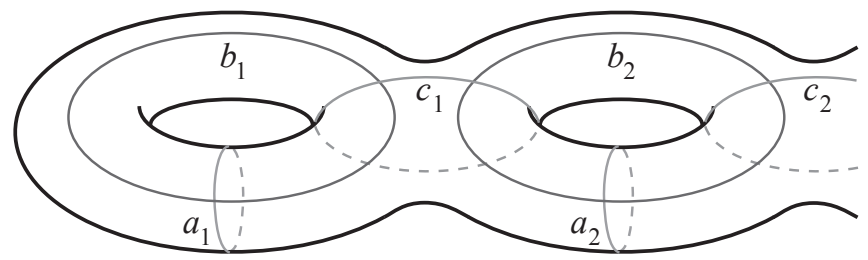

Figure 4. Bouillabaisse construction 


\section{$5 \quad$ Abundance}

We are tempted to say that most of the elements of $\operatorname{Mod}\left(S_{g}\right)$ are of pseudo-Anosov type. This intuition arises from what happens in genus 1: if we choose a "random" matrix in $\operatorname{Mod}\left(S_{1}\right)=\operatorname{SL}(2, \mathbb{Z})$, it has a strong probability of being hyperbolic (the absolute value of its trace is larger than 2). However, we need to precisely formulate the word "random" since all these groups are discrete groups.

A reasonable way to define this is to fix a set of generators of $\operatorname{Mod}\left(S_{g}\right)$ (for instance, the Dehn twists) and to look at bounded length words (or a ball of radius $N$ centred at the identity in the Cayley graph).

For some modular groups, and some generating sets, we can show that the proportion of pseudo-Anosov elements in the ball of radius $N$ tends exponentially fast to 1 as $N$ tends to infinity (see the work by Caruso-Wiest). There are also versions of this result using the tool of random walks.

\section{$6 \quad$ Counting}

Another way to show the abundance of pseudo-Anosov diffeomorphisms is to count them. Let us introduce

$$
\begin{aligned}
\mathcal{G}_{g}(T)=\{\text { conjugation classes of } \psi \mid \\
\psi \\
\psi \text { is pseudo-Anosov and } \log (\lambda(\psi))<T\} .
\end{aligned}
$$

Veech was the first to study the asymptotic behaviour of $\left|\mathcal{G}_{g}(T)\right|$ as $T$ tends to infinity. His work, starting in 1986, eventually culminated in the Eskin-Mirzakhani formula:

$$
\left|\mathcal{G}_{g}(T)\right| \sim_{T \rightarrow \infty} \frac{e^{(6 g-6) T}}{(6 g-6) T} .
$$

This formula was generalised later by Eskin-Mirzakhani-Rafi and Hamenstädt. The dynamical techniques that were employed used properties of the geodesic flow on the moduli space $\mathcal{M}_{g}$, inspired by the work of Margulis.

The key point is to make a parallel between the conjugacy class of $\psi$ and a closed curve on $\mathcal{M}_{g}$, the number $\log (\lambda(\psi))$ then being the length of this curve for some metric (the Teichmüller metric).

\section{$7 \quad$ Expansion factors}

Surprisingly, we do not know much about the expansion factors of pseudo-Anosov homeomorphisms.

\section{Realisations of algebraic numbers as expansion factors}

Looking at the action on homology (for a suitable cover), we easily deduce that $\lambda$ is an eigenvalue of a matrix with integer entries. It is thus an algebraic number (that is, the root of an irreducible polynomial $P \in \mathbb{Q}[X]$ ) of degree bounded by $3 g-$ 3. In fact, Thurston has shown that it is a bi-Perron number:

$$
\forall \alpha \neq \lambda, \lambda^{-1}, P(\alpha)=0 \Longrightarrow \lambda^{-1}<\alpha<\lambda .
$$

The converse (that is, if a bi-Perron number is an expansion factor) is an open problem. This is the subject of one of the last manuscripts of Thurston [9].

\section{Minimisation}

There are plenty of conjectures on this topic. The easiest ones to state are often about $\lambda$. For a fixed $g$, an easy argument that relates roots and coefficients shows that the set

$$
\operatorname{Spec}_{g}=\left\{\lambda(\psi), \psi: S_{g} \rightarrow S_{g} \text { is pseudo-Anosov }\right\} \subset \mathbb{R}
$$

is a discrete subset. What is its smallest element

$$
\delta_{g}=\min \left(\operatorname{Spec}_{g}\right) \text { ? }
$$

This is also an open problem! We know that $\delta_{1}=\frac{3+\sqrt{5}}{2}$ and $\delta_{2}=$ the largest root of $X^{4}-X^{3}-X^{2}-X+1 \simeq 1.72$ (compare with Example 4.2) but computing $\delta_{3}$ is already an open problem. It is not difficult to get an upper bound for $\delta_{g}$ (finding an example is sufficient). It is a little more subtle to get a lower bound. For all $g \geq 2$ :

$$
\frac{\log (2)}{6} \leq\left|\chi\left(S_{g}\right)\right| \cdot \log \left(\delta_{g}\right) \leq 2 \cdot \log \left(\frac{3+\sqrt{5}}{2}\right),
$$

where $\chi\left(S_{g}\right)=2-2 g$. We easily deduce that

$$
\limsup _{g \rightarrow \infty} g \log \left(\delta_{g}\right) \leq \log \left(\frac{3+\sqrt{5}}{2}\right) .
$$

McMullen conjectured that $\left(g \log \left(\delta_{g}\right)\right)_{g}$ converges but so far there is no proof of this. For a positive answer, one needs a better lower bound (on $g \log \left(\delta_{g}\right)$ ) than (1).

We present a recent result on matrices in this direction that was, surprisingly, not known before. McMullen [6] has shown that, for all $g \geq 1$, the smallest possible value of the spectral radius $\rho(A)$ of a primitive matrix $A \in \operatorname{Sp}_{2 g}(\mathbb{Z})$ (that is, one for which there exists $n$ such that all entries of $A^{n}$ are strictly positive) is given by the largest root of the polynomial

$$
X^{2 g}-X^{g}\left(1+X+X^{-1}\right)+1 .
$$

In particular, $\rho(A)^{g} \geq \frac{3+\sqrt{5}}{2}$. Even if this problem is closely related to the previous one, it does not (yet) provide a positive solution to the problem...

The discussions in the previous sections evoke a connection between these problems (of geometric nature) and the problem of minimising the eigenvalues of a matrix (of algebraic nature).

\section{Eigendirections of pseudo-Anosov homeomorphisms}

All the questions above are about eigenvalues of matrices (the expansion factor $\lambda$ ). What about the eigendirections associated to the eigenvectors? This is a very short section since we know almost nothing about it! It seems very difficult to characterise these directions at the moment, even if there are some partial results for genus 2 surfaces and Prym surfaces.

\section{Lonely guy conjecture}

If we choose a "random" flat metric $\omega$ on a surface $S_{g}$ (with respect to some probability measure on the moduli spaces), what kind of group of symmetries $\operatorname{SL}\left(S_{g}, \omega\right)$ could we expect? The answer that we guess is the trivial group. This is indeed the case (except perhaps if the surface has obvious nontrivial symmetry such as the hyperelliptic involution).

And now, what if we again choose a "random" flat metric $\omega$ among surfaces already having a symmetry? Again, the answer we expect is that generically the Veech group is cyclic. Surprisingly, this is not the case if the genus of $S_{g}$ is two! McMullen has given a quantitative version of this: the group $\mathrm{SL}\left(S_{g}, \omega\right)$ is very large. Its limit set is the full circle at infinity. 
What about when the genus $g$ is larger than three? This question is widely open. We conjecture that in general the group is (virtually) cyclic ...

\section{$9 \quad$ Suspensions and volumes}

There is a remarkable connection between the dynamics of pseudo-Anosov homeomorphisms in dimension two and the geometry in dimension three. The relation is given by the (very general) construction of suspension. To each $f: S_{g} \rightarrow$ $S_{g}$ we associate the 3 dimensional object

$$
M_{f}=S_{g} \times[0,1] /(1, x) \sim(0, f(x)) .
$$

Another famous theorem of Thurston states that $f=\psi$ is pseudo-Anosov if and only if $M_{\psi}$ is an hyperbolic 3-manifold. Thus it has a volume, although it is very hard to express it in terms of $\psi$. Kojima and McShane have recently established this beautiful inequality relating dynamic and geometric complexities:

$$
\log (\lambda(\psi)) \geq \frac{1}{3 \pi\left|\chi\left(S_{g}\right)\right|} \operatorname{vol}\left(M_{\psi}\right)
$$

where $\chi\left(S_{g}\right)=2-2 g$.

\section{To learn more about pseudo-Anosov maps}

The book by Fathi-Laudenbach-Poenaru [3] is a very good introduction to the topic, containing numerous details. It is based on the work of Thurston [8] on surface homeomorphisms. This book is also available in English.

The book by Farb-Margalit [4] is a more modern introduction to the modular group. It contains all the prerequisites and details for its study.

If one wants to learn more about pseudo-Anosov maps, the literature is rather vast. The recent works by Agol, Hironaka, Leininger and Margalit provide a nice "state of the art" and propose new approaches to the different problems alluded to above.

\section{References}

[1] Dmitri Anosov, Geodesic flows on compact riemannian manifolds of negative curvature, Proceedings of the Steklov Mathematical Institute 90 (1967), no. 1, 235

[2] Vladimir Arnold, Chapitres supplémentaires de la théorie des équations différentielles ordinaires, 1980.

[3] Albert Fathi, Fran, cois Laudenbach, and Valentin Poénaru, Travaux de thurston sur les surfaces, vol. 66, 1979.

[4] Benson Farb and Dan Margalit, A primer on mapping class groups, vol. 49, 2011.

[5] Étienne Ghys, Variations autour du théorème de récurrence de poincaré, J. de Maths des élèves de l'ÉNS Lyon 1 (1994).

[6] Curtis T. McMullen, Entropy and the clique polynomial, J. Topology 8 (2014), no. 1, 184-212.

[7] Stephen Smale, Differentiable dynamical systems, Bull. Amer. Math. Soc. 73 (1967), 747-817.

[8] William P. Thurston, On the geometry and dynamics of diffeomorphisms of surfaces, Bull. Amer. Math. Soc. 19 (1988), 417 431.

[9] __ Entropy in dimension one, ArXiV:1402.2008 (2008), 138

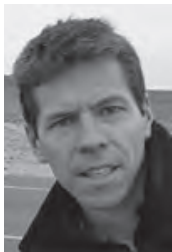

Erwan Lanneau is Professor of Mathematics at Grenoble University, France. He has been a researcher working at Institut Fourier, France, since 2012. His main research interests are in geometry, topology, dynamical systems and moduli spaces.

This is the English translation of the French article "Raconte moi un pseudo-Anosov", published in Gazette des Mathématiciens, Société Mathématique de France (No. 151, January 2017). The author thanks Fernando P. da Costa for the English translation. The author also thanks Pierre Dehornoy, Sébastien Gouëzel, Pascal Hubert, Vlad Sergiescu and Pierre Will for useful comments.

The EMS Newsletter is thankful to the Gazette des mathématicienes for authorisation to republish this text. 